For numbered affiliations see end of article.

heather.wardle@lshtm.ac.uk Cite this as: BMJ 2020;370:m2613 http://dx.doi.org/10.1136/bmj.m2613 Published: 1 July 2020

OPEN LETTER

\title{
Open letter from UK based academic scientists to the secretaries of state for digital, culture, media and sport and for health and social care regarding the need for independent funding for the prevention and treatment of gambling harms
}

Heather Wardle, ${ }^{1}$ James Banks, ${ }^{2}$ Paul Bebbington, ${ }^{3}$ Lindsey Blank, ${ }^{4}$ Henrietta Bowden Jones OBE, ${ }^{5}$ Stephanie Bramley, ${ }^{6}$ Christopher Bunn, ${ }^{7}$ Emma Casey, ${ }^{8}$ Rebecca Cassidy, ${ }^{9}$ Samuel R Chamberlain, ${ }^{10}$ James Close, ${ }^{11}$ Nathan Critchlow, ${ }^{12}$ Fiona Dobbie, ${ }^{13}$ Carolyn Downs, ${ }^{14}$ Simon Dymond, ${ }^{15}$ Emanuele Fino, ${ }^{16}$ Elizabeth Goyder, ${ }^{4}$ Cindy Gray, ${ }^{7}$ Mark Griffiths, ${ }^{16}$ Peter Grindrod, ${ }^{17}$ Lee Hogan, ${ }^{18}$ Alice Hoon, ${ }^{15}$ Kate Hunt, ${ }^{12}$ Richard James, ${ }^{19}$ Bev John, ${ }^{20}$ Jill Manthorpe, ${ }^{6}$ Jim McCambridge, ${ }^{21}$ David McDaid, ${ }^{22}$ Martin McKee, Sally McManus, ${ }^{23}$ Antony Moss, ${ }^{24}$ Caroline Norrie, ${ }^{6}$ David J Nutt, ${ }^{25}$ Jim Orford, ${ }^{6,26}$ Rob Pryce, ${ }^{4}$ Richard Purves, ${ }^{12}$ Gerda Reith, ${ }^{7}$ Amanda Roberts, ${ }^{27}$ Emmert Roberts, ${ }^{6}$ Gareth Roderique-Davies, ${ }^{20}$ Jim Rogers, ${ }^{27}$ Robert D Rogers, ${ }^{18}$ Stephen Sharman, 6, 28 John Strang, ${ }^{29}$ Richard Tunney, ${ }^{30}$ John Turner, ${ }^{28}$ Robert West, ${ }^{3}$ David Zendle 21

Dear secretaries of state,

As leading academic scientists studying gambling behaviours and its harms, we are writing to express our concern about the continuing support shown for the voluntary system of funding treatment, prevention and research in Great Britain. We feel compelled to write to you following the Betting and Gaming Council's (BGC) recent announcement (17 June 2020) that five of its operators will now allocate the long awaited increase in funding for prevention and treatment, first promised on 2 August 2019, to GambleAware rather than the charity Action Against Gambling Harms. Irrespective of which organisation funds are given to, the BGC's announcement exemplifies the longstanding weakness of a funding system that allows the gambling industry to regulate the availability and distribution of vital funds to address gambling harms across our communities. As we outline below, the continuance of this arrangement produces several negative effects that undermine the collective effort to reduce harms from gambling. It is also our belief that funds for research into gambling harms and their reduction should primarily be distributed through recognised independent organisations, such as UK Research and Innovation. We hereby urge you, as the secretaries of state with responsibilities for addressing gambling harms, to implement a statutory levy to fund effective prevention and treatment of gambling harms that is free both from industry influence and the perception of industry influence.

There is considerable concern that the existing system, whereby the gambling industry voluntarily provides funds for research, education and treatment, creates significant opportunities for them to influence this agenda. ${ }^{12}$ Deciding, unilaterally, who to fund is one way of exerting influence. The BGC announcement exemplifies this practice, where money promised to one charity was revoked at will and given to another, for reasons that have not been made public. This provides little assurance that the voluntary system is free from industry influence.

Delivering an effective strategy to reduce gambling harms requires surety and certainty of funding to enable effective planning and delivery of long term objectives. A voluntary system, reliant on the goodwill of the industry, is an inadequate way to develop such a system. Increases in funding first promised by five of the largest gambling operators nearly one year ago have yet to materialise, and industry has now demonstrated its ability and willingness to change the direction of funding at short notice. A system that contains such uncertainties is not suited to the long term development or delivery of a strategic plan to reduce harms.

Reducing harms requires a dual focus on treatment but also preventing harms from occurring in the first place. ${ }^{3}$ Prevention is a critical and central tenet of a public health based approach to harm reduction. Effective prevention requires independent assessment of what works and what doesn't to make recommendations for changes to policy and practice. Trust in the outcomes of such research by the public and policy makers is essential. There have been repeated critiques of studies produced under the existing voluntary system, undermining trust in research, outcomes, and expertise. ${ }^{124}$ The BGC announcement focuses on funding for treatment and says nothing about prevention. Equal attention needs to be given to preventing people from experiencing harms in the first place.

By offering a voluntary increase in funding, these operators clearly recognise the need for greater resources to tackle the harms they generate. We agree and believe a statutory levy is needed to address the inadequacies of the voluntary system to ensure that these promised increases in resources are delivered. There are clear benefits to doing so; it provides an opportunity to deliver harm reductions by ensuring a fair, independent, and trusted system for 
developing effective prevention activities. Effective prevention in turn delivers societal benefits through reductions in the social costs associated with gambling harms and a levy creates an equitable system by which all members of the industry contribute to addressing the harms they generate. We also believe that the funding for research raised by the statutory levy should be primarily awarded and administered independently through established bodies such as UK Research and Innovation and the National Institute for Health Research. This will ensure that research on gambling harms is sustainable for universities, attractive to the best researchers, and that policy can be based on the most robust evidence possible.

We urge you, the secretaries of state for digital, culture, media and sport and for health and social care, to review current funding arrangements and implement a statutory levy to deliver reductions in gambling harms.

\section{AUTHOR AFFILIATIONS}

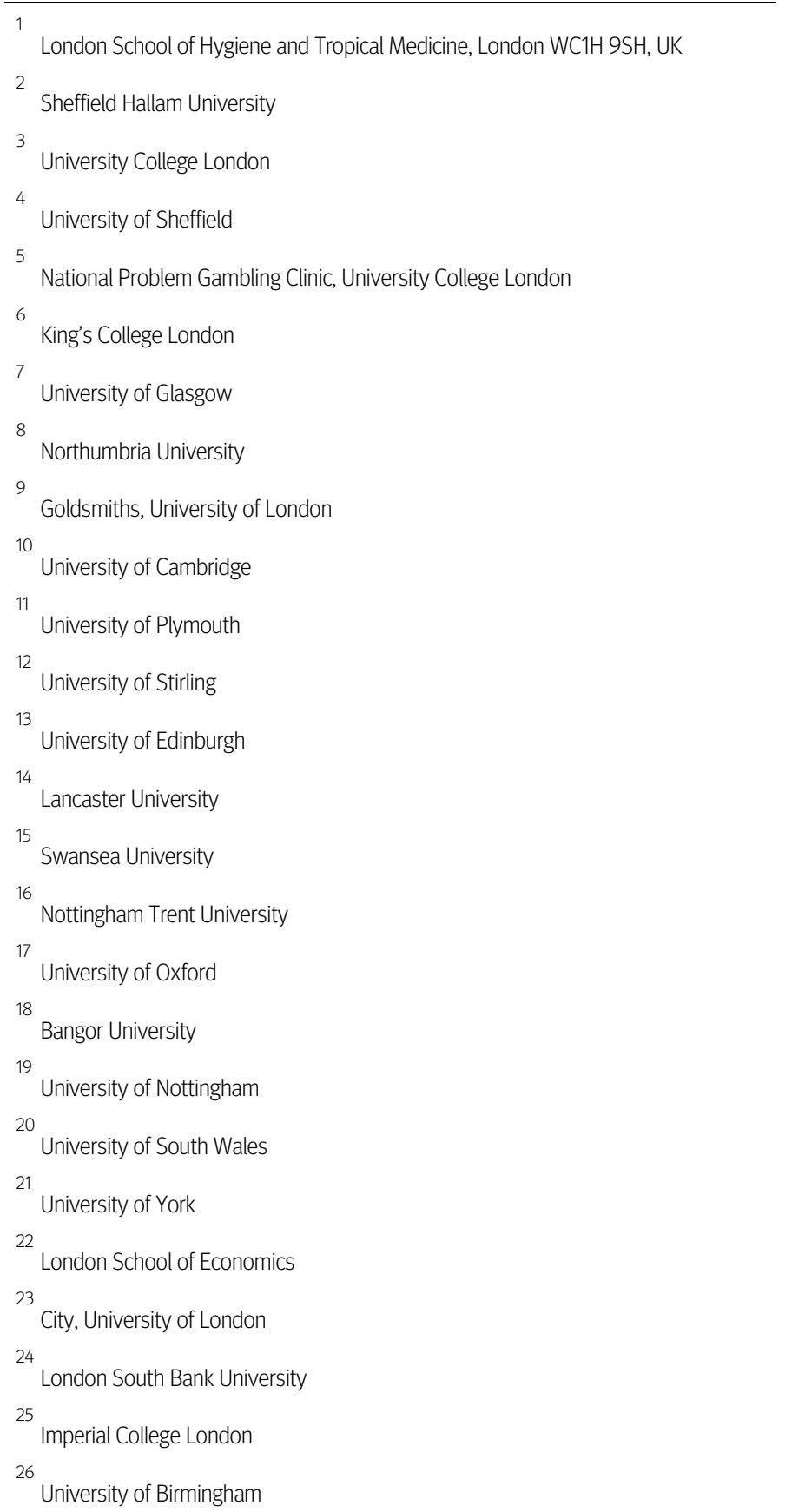

27 University of Lincoln

28 University of East London

29 National Addictions Centre, King's College London 30

Aston University

Competing interests: The following people are currently in receipt of or have previously received funding from GambleAware: HW, JC, JMa, CN, SB, MG, NC, SM, AM, JB, SD, AH, FD, RR, GR, RPu, and SS. The following individuals have these declarations: DM declares that the Care Policy and Evaluation Centre at the London School of Economics and Political Science has received funded for work that he undertook in 2019 for the Gambling Commission. GR declares funding from the Economic and Social Research Council (ESRC), the Medical Research Council, the Danish Research Council, the Scottish government, and the Responsibility in Gambling Trust (RiGT). Funding from RiGT was match funded and administered by the ESRC. She was previously a member of the Advisory Board for Safer Gambling. She has received honorarium from the Gambling Research Exchange Ontario, Alberta Gambling Research Institute, and the Gambling Commission. She is a member of the WHO panel on gambling and received reimbursements for travel and accommodation from them. $\mathrm{HB}$ ) is director of the National Problem Gambling Clinic, which is funded via the NHS and GambleAware. MG's university currently receives research funding from Norsk Tipping (the gambling operator owned by the Norwegian government). $M G$ has also received funding for several research projects in the area of gambling education for young people, social responsibility in gambling, and gambling treatment from GambleAware (formerly the Responsible Gambling Trust), a charitable body that funds its research programme based on donations from the gambling industry. MG also regularly undertakes consultancy for various gaming companies in the area of social responsibility in gambling. RC is a member of the WHO panel on gambling, which funds travel and accommodation for meetings. She has received travel and accommodation from the Alberta Gambling Research Institute. She was a special advisor to the House of Lords Select Committee Enquiry on the Social and Economic Impact of the Gambling Industry. SC consults for Promentis and receives stipends for editorial work at Elsevier (journals: Comprehensive Psychiatryand Neuroscience and Biobehavioural Reviews). AM acts as a paid consultant to the Safer Gambling Campaign Board. He is a current trustee for Alcohol Change UK. He previously acted as an unpaid scientific adviser to the Drinkaware Research and Impact Committee. JB supervises a doctoral student funded by GambleAware and has had expenses paid by the Alberta Gambling Research Institute. RJ received travel funding from the Swiss government (using public and academic funds) to attend and present at the 4th International Symposium on Excessive Gambling (Fribourg, Switzerland, $27-29$ June 2018) CD declares funding from People's Postcode Lottery and GamCare. GR-D declares funding from European Social Funds/Welsh government, Alcohol Concern (now Alcohol Change), research councils and the personal research budgets of several Welsh Senedd members. He is an invited observer (unpaid) of the cross party group on problem gambling at the Senedd Cymru Welsh Parliament and sits on the Beat the Odds steering group that is run by Cais Ltd (unpaid). He was previously a non-executive director of Solas-Cymru Ltd (now the Pobl Group), a third sector housing and social support organisation (unpaid). BJ has previously received funding from European Social Funds/Welsh government, Alcohol Concern (now Alcohol Change), research councils and the personal research budgets of several Welsh Senedd members. JT declares funding from Young Gamblers Education Trust and the Society for the Study of Addiction and supervision of a GambleAware PhD student. SS is funded by the Society for Study of Addiction and the National Institute for Health Research (NIHR). FD declares funding from RiGT, which was match funded and administered by the ESRC. RT declares funding from Camelot PLC. CN declares funding from GamCare. HW is currently funded by Wellcome, $\mathrm{NIHR}$, and ESRC for gambling research. Between 2015 and March 2020 she was funded by the Gambling Commission for her role on the Responsible Gambling Strategy Board/Advisory Board for Safer Gambling. She is a member of the WHO panel for gambling, which funded travel and accommodation at meetings. She has received travel and accommodation costs from the Alberta Gambling Research Institute. She has received payment for peer review from the Victorian Responsible Gambling Council. SB has received funding from Ridgeway Information Ltd, King's College London, the London School of Hygiene and Tropical Medicine, and GamCare in the past four years. She was previously employed by Citizens Advice Calderdale as a gambling support service trainer following GambleAware's partnership with Citizens Advice. RW has undertaken research and consultancy for companies that develop and manufacture smoking cessation medications (Pfizer, GSK, Johnson \& Johnson). All other authors have no conflicts to declare.

$1 \quad$ Orford J. The gambling establishment: challenging the system of the modern gambling industry and its allies. Routledge, 2019.

2 Cassidy R, Loussouarn C, Pisac A. Fair game: producing gambling research. The Goldsmiths report Goldsmiths, 2013. https://www.gold.ac.uk/media/documents-by-section/departments/anthropology/Fair-Game-Web-Final.pdf

3 Wardle H, Reith G, Langham E, Rogers RD. Gambling and public health: we need policy action to prevent harm. BMJ2019;365:11807. doi: 10.1136/bmj.l1807 pmid: 31068335

4 Cowlishaw S, Thomas SL. Industry interests in gambling research: Lessons learned from other forms of hazardous consumption. Addict Behav 2018;78:101-6. doi: 10.1016/j.addbeh.2017.11.007 pmid: 29136556 